\title{
Experimental demonstration of a four-port photonic crystal cross-waveguide structure
}

\author{
Yu, Yi; Heuck, Mikkel; Ek, Sara; Kuznetsova, Nadezda; Yvind, Kresten; Mørk, Jesper
}

Published in:

Applied Physics Letters

Link to article, DOI:

$10.1063 / 1.4772942$

Publication date:

2012

Document Version

Publisher's PDF, also known as Version of record

Link back to DTU Orbit

Citation $(A P A)$ :

Yu, Y., Heuck, M., Ek, S., Kuznetsova, N., Yvind, K., \& Mørk, J. (2012). Experimental demonstration of a fourport photonic crystal cross-waveguide structure. Applied Physics Letters, 101(25), 251113.

https://doi.org/10.1063/1.4772942

\section{General rights}

Copyright and moral rights for the publications made accessible in the public portal are retained by the authors and/or other copyright owners and it is a condition of accessing publications that users recognise and abide by the legal requirements associated with these rights.

- Users may download and print one copy of any publication from the public portal for the purpose of private study or research.

- You may not further distribute the material or use it for any profit-making activity or commercial gain

- You may freely distribute the URL identifying the publication in the public portal 


\section{AIP Applied Physics \\ Letters}

\section{Experimental demonstration of a four-port photonic crystal cross- waveguide structure}

Yi Yu, Mikkel Heuck, Sara Ek, Nadezda Kuznetsova, Kresten Yvind et al.

Citation: Appl. Phys. Lett. 101, 251113 (2012); doi: 10.1063/1.4772942

View online: http://dx.doi.org/10.1063/1.4772942

View Table of Contents: http://apl.aip.org/resource/1/APPLAB/v101/i25

Published by the American Institute of Physics.

\section{Related Articles}

Experimental realization of bending waveguide using anisotropic zero-index materials Appl. Phys. Lett. 101, 253513 (2012)

Mode latching and self tuning of whispering gallery modes in a stand-alone silica microsphere Appl. Phys. Lett. 101, 251105 (2012)

Ultralow $\mathrm{V} \pi \mathrm{L}$ values in suspended quantum well waveguides Appl. Phys. Lett. 101, 241111 (2012)

High performance broadband absorber in the visible band by engineered dispersion and geometry of a metaldielectric-metal stack

Appl. Phys. Lett. 101, 241116 (2012)

Broadband light-extraction enhanced by arrays of whispering gallery resonators Appl. Phys. Lett. 101, 241108 (2012)

\section{Additional information on Appl. Phys. Lett.}

Journal Homepage: http://apl.aip.org/

Journal Information: http://apl.aip.org/about/about_the_journal

Top downloads: http://apl.aip.org/features/most_downloaded

Information for Authors: http://apl.aip.org/authors

\section{ADVERTISEMENT}

\section{AIP Applied Physics Letters}

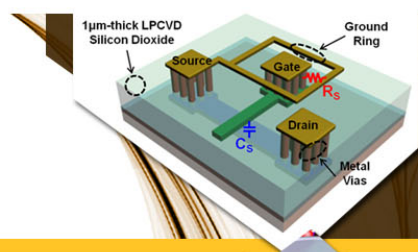

\section{SURFACES AND} INTERFACES

Focusing on physical, chemical, biological structural, optical, magnetic and electrical properties of surfaces and interfaces, and more..

\section{EXPLORE WHAT'S NEW IN APL}

SUBMIT YOUR PAPER NOW!
ENERCY CONVERSION AND STORACE 


\title{
Experimental demonstration of a four-port photonic crystal cross-waveguide structure
}

\author{
Yi Yu, ${ }^{a)}$ Mikkel Heuck, Sara Ek, Nadezda Kuznetsova, Kresten Yvind, and Jesper Mørk \\ DTU Fotonik, Technical University of Denmark, DK-2800 Kongens Lyngby, Denmark
}

(Received 20 October 2012; accepted 6 December 2012; published online 20 December 2012)

\begin{abstract}
We report the design and fabrication of a four-port InP photonic crystal cavity-waveguide structure in which two crossing waveguides intersect in a cavity. Transmission measurements show that by exploiting mode-gap effects, high cross-talk suppression between the two waveguides can be obtained. In addition, the waveguides couple to two distinct cavity resonances with different quality-factors as well as small mode volumes. This structure is promising for realizing ultra-fast, low-energy optical switches or memories. (C) 2012 American Institute of Physics.
\end{abstract}

[http://dx.doi.org/10.1063/1.4772942]

Photonic crystal $(\mathrm{PhC})$ membrane structures enable the realization of waveguides with tailored dispersion as well as nanocavities, which can be used to control and enhance light-matter interactions, with applications in integrated photonics $^{1-3}$ and quantum information technology. ${ }^{4}$ Featuring small modal volume and large quality-factor (Q-factor), PhC nanocavity devices may enhance nonlinearities due to Kerr or carrier effects, ${ }^{5-8}$ thus realizing ultra-compact and integrated devices facilitating ultra-fast, low-energy optical signal processing.

For switching applications, such as de-multiplexing, ${ }^{9}$ the high-speed time-multiplexed data signal is switched by a control signal at the frequency of the tributary. Since the two signals need to be separated at the output, separate channels are required for the two beams, either enabled by wavelength or spatially using a three- or four-port structure. ${ }^{10}$ However, in most previous $\mathrm{PhC}$ device demonstrations, two-port structures were considered, consisting of a single mode cavity coupled with a single line-defect waveguide acting as input as well as output channel..$^{5-7}$ In those cases, the control and data signals are injected into a common input waveguide simultaneously ${ }^{6}$ and interact with the same cavity resonance. The weak data signal is switched on or off through the cavity resonance shift caused by the strong control signal. This structure has several disadvantages. In order to distinguish the signals at the output, optical band-pass filters need to be used. ${ }^{5,7}$ This causes additional complexity of the system and for a minimized switching energy, the optimum operation wavelengths of the data and control signal may not be compatible with the required wavelength separation needed for the band-pass filtering and a compromise must be made. Besides, the single resonance of the structure does not allow for optimum cavity enhancement of both the control and data signal, which may differ both in wavelength and bandwidth. This becomes a critical issue if the criteria on energy consumption are to be met at high speeds. ${ }^{11}$ In this paper, we experimentally demonstrate a structure, where the two signals excite different, yet spatially overlapping, resonances and are spatially separated at the output.

\footnotetext{
a)Electronic mail: yiyu@fotonik.dtu.dk.
}

Designs for low cross-talk multi-port structures with crossing waveguides have been suggested previously and numerically investigated. ${ }^{12,13}$ Experimental results were obtained for a square lattice geometry ${ }^{14}$ but not for a triangular lattice, which is favored for ultra-compact cavities and slow light structures. ${ }^{15}$ The structure investigated here, shown schematically in Fig. 1(a), is a four-port PhC slab similar to the multi-arm structure in Ref. 13. It has a defecttype single cell nanocavity coupled with two waveguides, WG1 and WG2, crossing each other at angles of $60^{\circ}$ or $120^{\circ}$. The cavity is formed by removing one hole in the center and displacing 18 neighboring holes by the amounts $A_{i}$ or $B_{i}(i=1,2,3)$. The single line-defect waveguides WG1 and WG2 are based on normal W1 waveguides (defined as

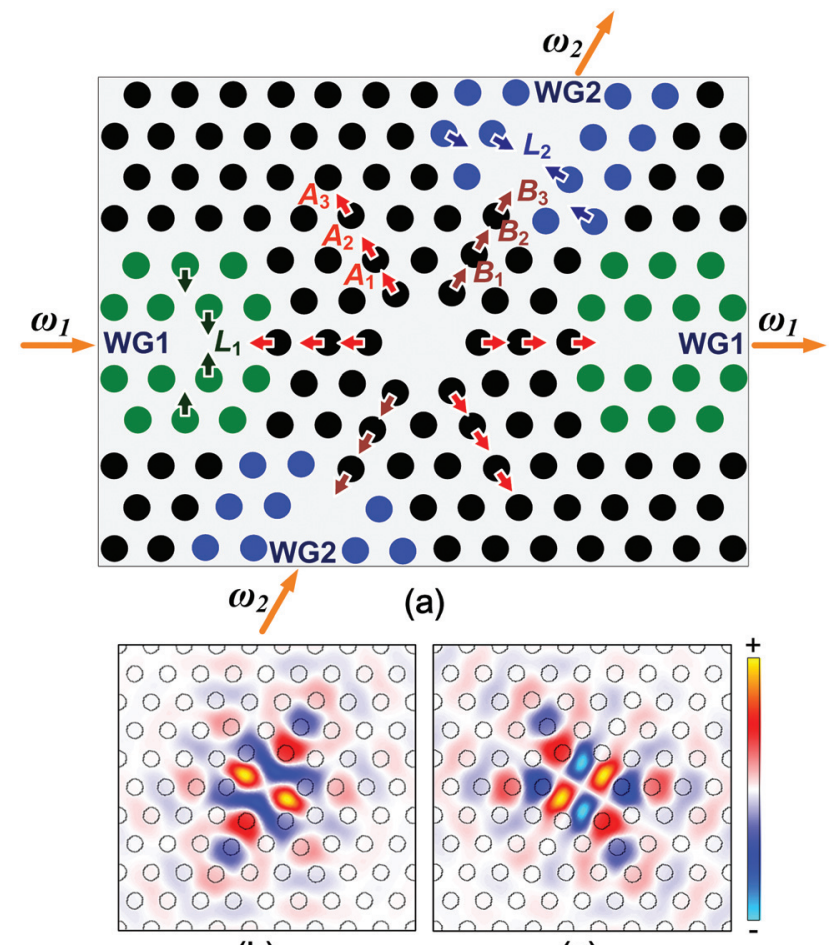

(b)

(c)

FIG. 1. (a) Schematic of the four-port structure with two crossing waveguides, WG1 and WG2, intersecting in a single cell cavity. Magnetic field intensity of (b) even and (c) odd quadrupole modes of the single cell cavity. 
the removal of one row of air holes) but their innermost two rows of holes (green and blue holes) are shifted a distance $L_{i}(i=1,2)$. The air hole shifting directions are indicated by the arrows in Fig. 1(a).

If the structure is symmetric $\left(A_{i}=B_{i}, L_{1}=L_{2}\right)$, the single cell cavity has $\mathrm{C}_{6 \mathrm{v}}$ symmetry, ${ }^{16}$ resulting in the existence of a nondegenerate hexapole and monopole mode, and a pair of doubly degenerate dipole and quadrupole modes. Our investigations only focus on the quadrupole modes. Figs. 1(b) and 1(c) show the magnetic field intensity of the corresponding even and odd modes. If the cavity mode frequencies are commensurate with WG1 and WG2 propagating waveguide modes, light injected into either WG1 or WG2 is expected to couple into both WG1 and WG2. In order to suppress crosscoupling between the waveguides we exploit the mode-gap effect, ${ }^{13}$ i.e., the modes of WG1 and WG2 are separated in frequency and made to couple to different cavity resonances, as illustrated in the calculated band diagram in Fig. 2(a). The first is achieved by choosing different waveguide parameters $\left(L_{1} \neq L_{2}\right)$ and the latter by making the cavity asymmetric $\left(A_{i}\right.$ $\neq B_{i}$ ). Figs. 2(b) and 2(c) show the field intensity maps, calculated using three dimensional finite-difference time-domain (3D FDTD) technique, of such an asymmetric structure when exciting either WG1 or the WG2 mode at the input, illustrating that light can pass through the cavity from either waveguide without crosstalk.

The detailed design procedure is the following: starting from the symmetric structure, by shifting the innermost two rows of holes of WG2 away from the waveguide center, i.e., decreasing the value of $L_{2}$ to make $L_{2}<L_{1}$, the fundamental and second WG2 waveguide modes (solid and hollow red markers) move to lower frequency and separate from the

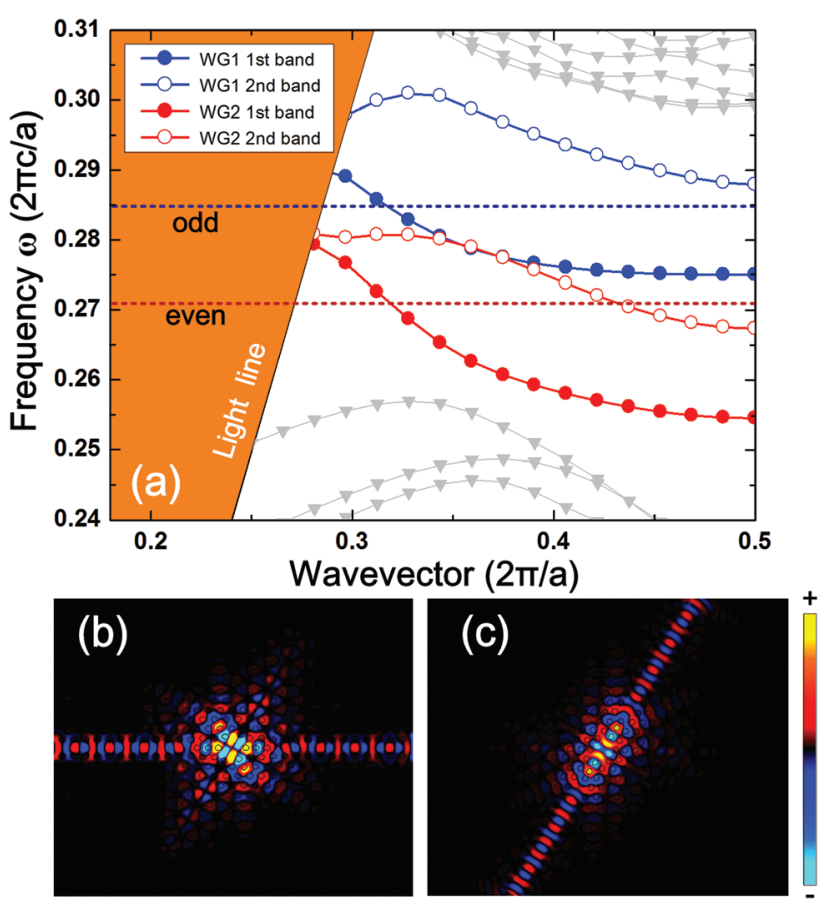

FIG. 2. (a) Band diagram of an asymmetric four-port structure calculated using plane wave expansion method. (b) Magnetic field intensity of cavity odd mode only coupling into WG1 fundamental mode. (c) Magnetic field intensity of cavity even mode only coupling into WG2 fundamental mode. fundamental and second WG1 modes (solid and hollow blue markers) as shown in Fig. 2(a). Moving the 6 holes neighboring the cavity away from the center, i.e., increasing the values of $B_{i}$ to make $B_{i}>A_{i}$, the cavity even mode (horizontal dashed red) can also be shifted down below the odd mode (horizontal dashed blue) in frequency, without affecting the cavity odd mode significantly. This is because the field intensity is much larger near these 6 holes with $B_{i}$ shifts for the even mode than for the odd mode, as can be seen from Figs. 1(b) and 1(c). Consequently, the leakage from WG1 (WG2) to WG2 (WG1) is suppressed.

In the actual design, we have used parameters appropriate for InP membranes with refractive index $n$ of 3.16, hole radius $r$ of $0.259 a$, and membrane thickness $h$ of $0.77 a$, where $a$ is the lattice constant. In contrast to the design of Ref. 13, we have restricted ourselves to the use of one common hole size in order to increase the robustness towards fabricationrelated uncertainties. By merely changing the relative positions of the holes, it is still possible to engineer both the dispersion of the waveguide modes as well as the cavity resonances and their Q-factors to realize the desired functionality. A disadvantage of using only one hole size, however, is that the waveguides become multi-mode, ${ }^{17}$ considering the cavity odd mode and the WG2 second mode has the same mode symmetry, the cavity odd mode frequency thus needs to be sufficiently high to be non-overlapping with the higherorder WG2 mode. The specific parameters for the design are $A_{1}=0.18 a, A_{2}=0.04 a, A_{3}=0.04 a, B_{1}=0.42 a, B_{2}=0.34 a$, $B_{3}=0.06 a$, and $L_{1}=0.12 a, L_{2}=-0.08 a$ (minus means moving the hole away from the center of the waveguide).

The photonic crystal pattern is first defined in positive resist (ZEP520A) by electron-beam lithography and then transferred to a $200 \mathrm{~nm} \mathrm{Si}_{3} \mathrm{~N}_{4}$ hard mask by $\mathrm{CHF}_{3} / \mathrm{O}_{2}$ reactive-ion etching (RIE). After removing the resist, the pattern was transferred to a $340 \mathrm{~nm}$ InP layer by a cyclic $\mathrm{CH}_{4} / \mathrm{H}_{2}$ and $\mathrm{O}_{2}$ RIE. The $\mathrm{Si}_{3} \mathrm{~N}_{4}$ layer is removed by $\mathrm{HF}$ and finally the membrane structure is formed with diluted $\mathrm{H}_{2} \mathrm{SO}_{4} / \mathrm{H}_{2} \mathrm{O}_{2}$ selective wet-etching to remove a $1 \mu \mathrm{m} \mathrm{In}_{0.53} \mathrm{Ga}_{0.47} \mathrm{As}$ sacrificial layer located below the membrane.

Fig. 3 shows scanning electron microscope (SEM) images of one of the fabricated devices, for which $a=440 \mathrm{~nm}$, $r=114.9 \mathrm{~nm}$. In order to facilitate external coupling, the access waveguides of the $900 \mu \mathrm{m}$ long structure are equipped with mode adapters ${ }^{18}$ through which only the fundamental waveguide mode can be excited by a single mode fiber due to the even symmetry of the injection condition. Two broadband $60^{\circ}$ bendings along WG 2 are implemented, c.f. Fig. 3. The bending structure, similar to the designs in Ref. 19, is realized by connecting two air holes with an arc of $60^{\circ}$ to reduce the light leakage. 3D FDTD simulations predict that the cavity odd mode coupling with WG1 has a resonance peak at $1531.2 \mathrm{~nm}$ and a mode volume of $0.65(\lambda / n)^{3}$; the cavity even mode coupling with WG2 has a resonance peak at $1601.6 \mathrm{~nm}$ and a larger mode volume of $0.77(\lambda / n)^{3}$.

The fabricated devices are characterized using a tunable continuous wave (CW) laser source. Input light is TEpolarized (electric field in the plane of the $\mathrm{PhC}$ slab) utilizing a polarization controller and is coupled in and out of the device using lensed single mode fibers. The output is measured by an optical spectrum analyzer, while an infrared camera 


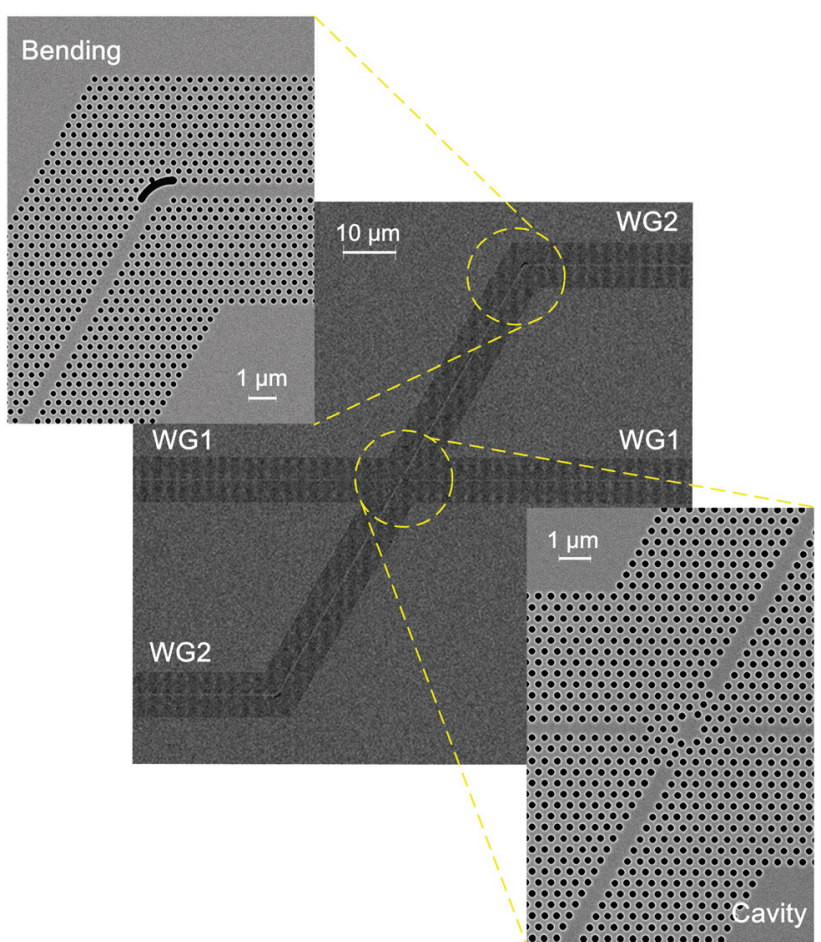

FIG. 3. SEM images of fabricated four-port InP crossing-waveguide structure. Two waveguides WG1 and WG2 intersect in a cavity.

(Xeva-1.7-320) is mounted on a microscope to image the far-field scattered light from the top of the sample.

Injecting light into WG1, Fig. 4(a) shows the normalized transmission spectra from WG1 input to WG1 output (WG1WG1) and from WG1 input to WG2 output (WG1-WG2). Here, the spectra of WG1-WG1 shows a resonance with a peak centered at $1531.5 \mathrm{~nm}$ and a Q-factor of $\sim 8.21 \times 10^{3}$ as obtained by fitting the resonance with a Lorentzian lineshape. The infra-red images, Figs. 4(b) and 4(c), clearly show the transmission of the beam through WG1 onresonance and from Fig. 4(a) we see that, on-resonance, the coupling from WG1 to WG2 is suppressed by more than $30 \mathrm{~dB}$. The transmission loss including coupling and propagation losses for WG1 at the resonance is about $-34 \mathrm{~dB}$.

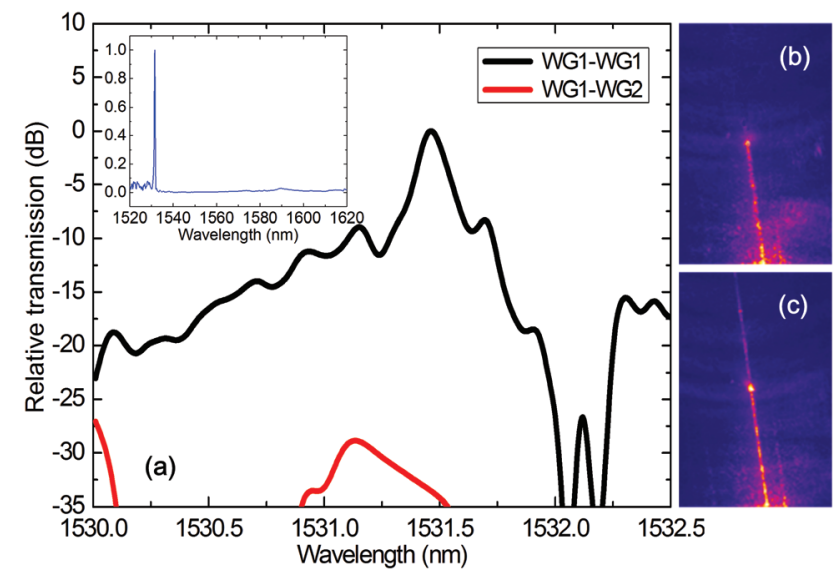

FIG. 4. (a) Transmission spectra from WG1 input to WG1 output (black), and from WG1 input to WG2 output (red). Inset shows the normalized transmission spectra from WG1 input to WG1 output in linear scale from 1520 to $1620 \mathrm{~nm}$. Image from infrared camera when the cavity is (b) off-resonance and (c) on-resonance. Light is injected from the bottom.

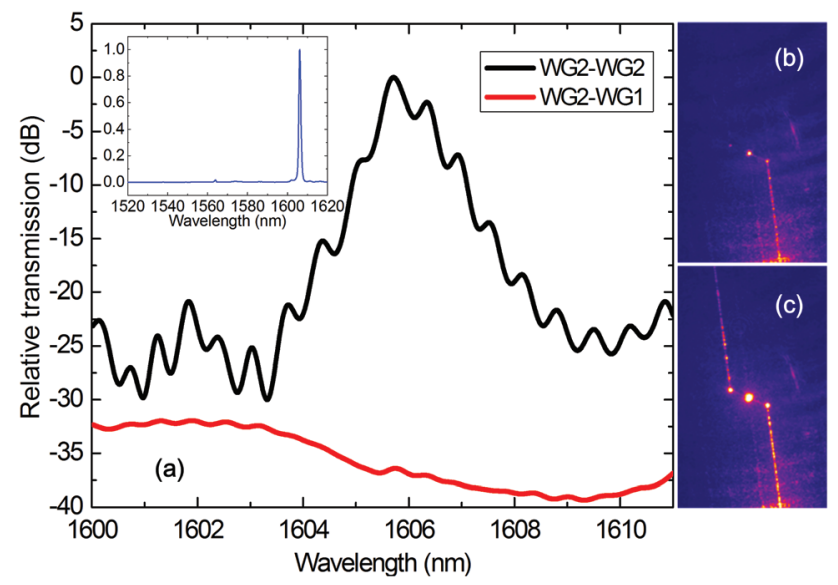

FIG. 5. (a) Transmission spectra from WG2 input to WG2 output (black), and from WG2 input to WG1 output (red). Inset shows the normalized transmission spectra from WG2 input to WG2 output in linear scale from 1520 to $1620 \mathrm{~nm}$. Image from infrared camera when the cavity is (b) off-resonance and (c) on-resonance. Light is injected from the bottom.

From 3D FDTD simulations, this relatively low transmission is mainly caused by a low cavity transmission which is originated from a much lower out-of-plane Q-factor of the cavity odd mode compared with its in-plane Q-factor. ${ }^{20,21}$ This can be improved by gradually tapering the size of the 3 displaced cavity holes with $A_{i}$ shifts along WG1 to make the evanescent field tunnel more gently between the cavity and WG1. ${ }^{22}$ In addition, the narrow waveguide width of WG1 may also increase the loss.

Injecting light into WG2, Fig. 5(a) shows the normalized transmission spectra from WG2 input to WG2 output (WG2WG2) and from WG2 input to WG1 output (WG2-WG1). The WG2-WG2 transmission has a resonance centered at $1605.7 \mathrm{~nm}$ and a Q-factor of $\sim 2.8 \times 10^{3}$. From Figs. 5(b) and $5(c)$, we see that when the cavity is on-resonance, light only propagates in WG2, and from Fig. 5(a), the leakage into WG1 is suppressed by more than $36 \mathrm{~dB}$. The transmission loss for WG2 at the resonance is about $-15 \mathrm{~dB}$.

From the insets of Figs. 4 and 5, only one resonance can be observed. This agrees with the simulation results in which the dipole and monopole modes of the single cell cavity have their resonances far beyond the L-band. The hexapole mode centered at $\sim 1565 \mathrm{~nm}$ does not couple into WG1 nor WG2. This is because its mode frequency lies just above the top edge of the WG2 second band diagram in this design, and it has a different mode symmetry compared with the WG1 fundamental waveguide mode. ${ }^{23-25}$

In conclusion, a multi-mode $\mathrm{InP} \mathrm{PhC}$ cavity structure has been designed and fabricated. The structure consists of two crossing waveguides intersecting in a single cell cavity. Using mode-gap effects, the leakage between the waveguides was suppressed by more than $30 \mathrm{~dB}$. The two waveguides couple to two different resonances, whose frequencies both is within the $\mathrm{C}+\mathrm{L}$ band and their quality-factors can be independently tuned. The structure was realized using a single hole size, which is advantageous from a fabrication point of view. In the fabricated structure, quality factors of $8.2 \times 10^{3}$ and $2.8 \times 10^{3}$ were demonstrated. This makes the structure very suitable for optical signal processing tasks such as all-optical de-multiplexing, where the control and data signals may have different 
wavelengths and bandwidths, and where demands on energy consumption per bit require separate optimization of the resonances. In addition, the cavity modes have small mode volumes which are promising for fast data-processing. Though this structure is an air suspended $\mathrm{PhC}$ having low thermal conductivity, we expect thermal effects can be reduced using such as selective regrowth technique ${ }^{7}$ or encapsulating the structure in $\mathrm{SiO}_{2} .{ }^{26}$

The authors acknowledge financial support from Villum Fonden via the NATEC (NAnophotonics for TErabit Communications) Centre. The authors would also like to thank Pierre Colman for help with the experimental set up and fruitful discussions.

${ }^{1}$ T. Tanabe, M. Notomi, S. Mitsugi, A. Shinya, and E. Kuramochi, Opt. Lett. 30, 2575 (2005).

${ }^{2}$ T. F. Krauss, J. Phys. D: Appl. Phys. 40, 2666 (2007).

${ }^{3}$ M. Notomi, A. Shinya, K. Nozaki, T. Tanabe, S. Matsuo, E. Kuramochi, T. Sato, H. Taniyama, and H. Sumikura, IET Circuits Devices Syst. 5, 84 (2011).

${ }^{4}$ J. Vuckovic and Y. Yamamoto, Appl. Phys. Lett. 82, 2374 (2003).

${ }^{5}$ K. Nozaki, T. Tanabe, A. Shinya, S. Matsuo, T. Sato, H. Taniyama, and M. Notomi, Nature Photon. 4, 477 (2010).

${ }^{6}$ C. Husko, A. De Rossi, S. Combrié, Q. Vy Tran, F. Raineri, and C. W. Wong, Appl. Phys. Lett. 94, 021111 (2009).

${ }^{7}$ K. Nozaki, A. Shinya, S. Matsuo, Y. Suzaki, T. Segawa, T. Sato, Y. Kawaguchi, R. Takahashi, and M. Notomi, Nature Photon. 6, 248 (2012).

${ }^{8}$ A. De Rossi, M. Lauritano, S. Combrié, Q. Vy Tran, and C. Husko, Phys. Rev. A 79, 043818 (2009).
${ }^{9}$ T. Yamamoto, E. Yoshida, and M. Nakazawa, Electron. Lett. 34, 1013 (1998).

${ }^{10}$ M. F. Yanik, S. Fan, M. Soljacic, and J. D. Joannopoulos, Opt. Lett. 28, 2506 (2003).

${ }^{11}$ M. Heuck, P. T. Kristensen, and J. Mørk, Opt. Express 19, 18410 (2011).

${ }^{12}$ S. G. Johnson, C. Manolatou, S. Fan, P. R. Villeneouve, J. D. Joannopoulos, and H. A. Haus, Opt. Lett. 23, 1855 (1998).

${ }^{13}$ S. H. Kwon, M. Kamp, A. Forchel, M. K. Seo, and Y. H. Lee, Opt. Express 15, 11399 (2008).

${ }^{14}$ Y.-G. Roh, S. Yoon, H. Jeon, S. H. Han, and Q.-H. Park, Appl. Phys. Lett. 85, 3351 (2004).

${ }^{15}$ B.-S. Song, S. Noda, T. Asano, and Y. Akahane, Nature Mater. 4, 207 (2005).

${ }^{16}$ S. H. Kim and Y. H. Lee, IEEE J. Quantum Electron. 39, 1081 (2003).

${ }^{17}$ A. Adibi, R. K. Lee, Y. Xu, A. Yariv, and A. Scherer, Electron. Lett. 36, $1376(2000)$.

${ }^{18}$ Q. V. Tran, S. Combrié, P. Colman, and A. De Rossi, Appl. Phys. Lett. 95, 061105 (2009).

${ }^{19}$ K. Lenglé, M. Gay, L. Bramerie, J. C. Simon, S. Combrié, G. Lehoucq, A. de Rossi, S. Malaguti, G. Bellanca, and S. Trillo, IEEE Photon. Technol. Lett. 24, 2109 (2012).

${ }^{20}$ M. Notomi, A. Shinya, S. Mitsugi, E. Kuramochi, and H.-Y. Ryu, Opt. Express 12, 1551 (2004).

${ }^{21}$ Y. Akahane, T. Asano, B.-S. Song, and S. Noda, Opt. Express 13, 1202 (2005).

${ }^{22}$ K. Srinivasan and O. Painter, Opt. Express 11, 579 (2003).

${ }^{23}$ G. H. Kim, Y. H. Lee, A. Shinya, and M. Notomi, Opt. Express 12, 6624 (2004).

${ }^{24}$ S. K. Kim, G. H. Kim, S. H. Kim, Y. H. Lee, S. B. Kim, and I. Kim, Appl. Phys. Lett. 88, 161119 (2006).

${ }^{25}$ T. Baba, D. Mori, K. Inoshita, and Y. Kuroki, IEEE J. Sel. Top. Quantum Electron. 10, 484 (2004).

${ }^{26}$ Y. Halioua, A. Bazin, P. Monnier, T. J. Karle, G. Roelkens, I. Sagnes, R. Raj, and F. Raineri, Opt. Express 19, 9221 (2011). 